\title{
Cutaneous Metastasis in a Previously Known Case of Ovarian Dysgerminoma: A Case Report
}

\author{
Farokh Seilanian Toosi (iD) ${ }^{1}$, Malihe Hasanzadeh ${ }^{2}$, Mona Maftouh (iD ${ }^{1}$ and Ahmadreza Tavassoli (iD) ${ }^{1,}$ \\ ${ }^{1}$ Radiology Department, Mashhad University of Medical Sciences, Mashhad, Iran \\ ${ }^{2}$ Obstetrics and Gynecologic Surgery Department, Mashhad University of Medical Sciences, Mashhad, Iran \\ "Corresponding author: Radiology Department, Mashhad University of Medical Sciences, Mashhad, Iran. Email: tavassoli.art@gmail.com
}

Received 2020 May 09; Revised 2021 September 12; Accepted 2021 September 15.

\begin{abstract}
Introduction: Cutaneous metastasis is an uncommon manifestation that mostly occurs in patients with previous gastrointestinal or gynecological malignancies. Local recurrence usually can be seen in surgical incisions. Dysgerminomas are rare ovarian tumors that usually have local invasions, but metastases are infrequent. Here, we described a case of dysgerminoma and cutaneous metastasis in the surgical incision.

Case Presentation: The patient was a 39-year-old woman previously known as ovarian dysgerminoma, who had undergone surgery one year ago. Ultrasound examination a year after surgery showed a $35 \times 33$ millimeters hypoechoic solid tumor in the left ovary and another 3 masses with similar echo pattern and vascularity were found in the surgical incision. Magnetic resonance imaging (MRI) findings were compatible with cutaneous metastasis of pervious dysgerminoma. Pathologic evaluation and Immunohistochemistry (IHC) confirmed dysgerminoma's skin metastasis. She received chemotherapy due to the recurrence of malignancy. Conclusions: Local recurrence and metastases (even in the skin) should be kept in mind and the clinician must examine the surgical site skin in patients with a history of malignancy.
\end{abstract}

Keywords: Ovarian Neoplasms, Dysgerminoma, Recurrence, Neoplasm Metastasis

\section{Introduction}

Recurrence in gastrointestinal and gynecological malignancies can be presented with cutaneous metastases which usually occur in the incisional scars. The incidence of cutaneous metastasis in internal malignancies has been reported from 1 to $4.6 \%$ (1). The most common malignancies which metastasize to the skin are breast and ovarian tumors in women and lung and colon cancers in men (2). In the case of laparoscopic surgeries, recurrence can be found in the laparoscopic port scars. Port site metastases are found in 1 to $2 \%$ of laparoscopic surgeries (3).

Cutaneous metastases may be the first presentation of malignancy in rare cases $(4,5)$. Cell implants in surgical scars also can be seen in non-invasive neoplasms such as borderline ovarian tumors (6) or even benign lesions, eg, endometriosis in $1 \%$ of cases (7). Direct implantation of tumor cells is the most common mechanism in surgical scar metastases (8).

In the differential diagnosis, muscle lymphoma, sarcoma, and benign lesions like muscle hemangioma, in- tramuscular ganglia, and myxoma must be considered. Magnetic Resonance Imaging (MRI) could help distinguish these, though pathologic specimens are essential for ultimate diagnosis (9).

The most common ovarian tumor which metastasizes to the skin is epithelial adenocarcinoma (serous, mucinous, and endometrioid) (10). Dysgerminoma is the most common ovarian germ cell tumor that may have a local invasion, lymphatic spread to pelvic lymph nodes, or hematogenous metastases (11). However, cutaneous metastases are relatively rare but they are of important clinical significance.

Here, we presented a case of dysgerminoma and cutaneous metastasis in the surgical incisions.

\section{Case presentation}

The patient was a 39-year-old woman who had one child. She presented with abdominal pain and ultrasound examinations showed a large mass $(160 \times 150 \mathrm{~mm})$ lateral 
to the uterus. The radiologists suggested a huge pedunculated leiomyoma of the uterus as a differential diagnosis.

The patient underwent surgery with a possible diagnosis of leiomyoma. A tumoral lesion originating from the left ovary was observed. The abdominal cavity was explored and there was no obvious involvement, so the tumoral lesion and the left ovary were totally removed and multiple biopsies of the left fallopian tube, right ovary, omentum, posterior wall of the bladder, and mesocolon were obtained which all were free of tumor. Pathologic evaluation of the tumor revealed dysgerminoma of the ovary.

Regarding the patient's age, childbearing desire, and lack of tumor spread to the other organs, fertilitypreserving surgery performed for the patient was enough and the patient had not received postoperative chemotherapy. Following up with tumor markers and imaging were recommended. Three months after surgery, the laboratory data revealed Alpha-fetoprotein of $0.99 \mathrm{IU} / \mathrm{mL}$ (normal range: 0 - $2 \mathrm{IU} / \mathrm{mL}$ ), Lactate dehydrogenase (LDH) $250 \mathrm{U} / \mathrm{L}$ (normal range: up to $480 \mathrm{U} / \mathrm{L}$ ), CA-125 $4.51 \mathrm{U} / \mathrm{mL}$ (normal range: up to 35 ), and Beta hCG $<2$ IU/L (Negative).

6 months post-operative follow-up did not show recurrence and residual remnants. One year after surgery, the ultrasound examination showed a $35 \times 33$ millimeters hypoechoic solid tumor in the left adnexa with internal vascularity in Doppler examination. Also, another 3 masses with similar echo pattern and vascularity were found in the surgical incision. All other examinations were normal. MRI was performed for further evaluation and the findings were compatible with local recurrence and cutaneous metastasis of pervious dysgerminoma (Figures 1-5).

Core Needle Biopsy of surgical scar lesion was performed and the sections showed muscles and fibroconnective tissues with malignant neoplastic cells which were suggestive for metastatic lesions (Figure 6) and Immunohistochemistry (IHC) study for CK, SALL4, and HPL was recommended. The final diagnosis based on IHC findings was surgical site metastasis of dysgerminoma. After that, the patient received 4 courses of chemotherapy regimens consisting of bleomycin, etoposide, and platinum (BEP).

\section{Discussion}

Ovarian neoplasms are categorized into 3 groups: epithelial, sex cord, and germ cells. Dysgerminoma is an ovarian tumor consisting of primitive and undifferentiated germ cells. Most of them are benign lesions and only $3 \%$ are malignant (11). Although dysgerminoma is a chemotherapy-sensitive ovarian tumor, in the case of young women with early stages of dysgerminoma, unilateral salpingo-oophorectomy is recommended as a treatment of choice, particularly in the cases in which fertility is important. But the relapse rate is about $10 \%$ to $20 \%$ (11, 12).

Ovarian cancers are often associated with metastasis. They can occur with the direct spread of malignant cells within the abdominal cavity or via lymphatic vessels (13). Although pleura, liver, bone, lung, and lymph nodes are common sites of distant metastasis, cutaneous metastases are rare in ovarian malignancies (10). Nowadays the main treatment for advanced stages of dysgerminoma, eg, disease with extra-ovarian spread, is post-operative adjuvant chemotherapy with carboplatin or cisplatin in combination with etoposide and bleomycin (14).

Patients with skin metastasis are usually those who did not receive postoperative chemotherapy or were resistant to chemotherapy (8). Recently skin metastases prevalence is increased because of the patient's survival improvements.

Cutaneous metastases have 2 subtypes: Umbilical metastases -also known as Sister Joseph Nodules (SJNs) which are related to peritoneal metastases and indicate a more advanced tumor; however it can be the first manifestation of the tumor in some patients (13) and the other type is Non-SJN, which usually occurs in surgical sites and the most common place is the abdominal wall $(13,15)$. One of the prone places is surgical incision especially laparoscopic ports $(13,16)$. Tumor cells are extended by direct implantation or via hematogenous pathways through surgery or injury by taking advantage of wound healing processes that precipitate tumor growth. Also, they can spread to lymph node vicinities after lymphadenopathy (8).

In this case presentation, we reported a rare case of incisional skin metastasis in ovarian dysgerminoma. Our patient had undergone unilateral oophorectomy without receiving chemotherapy and scar incision recurrence in our patient might be due to not taking the adjuvant chemotherapy.

\subsection{Conclusions}

Despite the low risk of malignancy in dysgerminoma, follow-up evaluation is of great importance particularly in the cases who had not received chemotherapy. Local recurrence and metastases (even in the surgical site skin) are the 


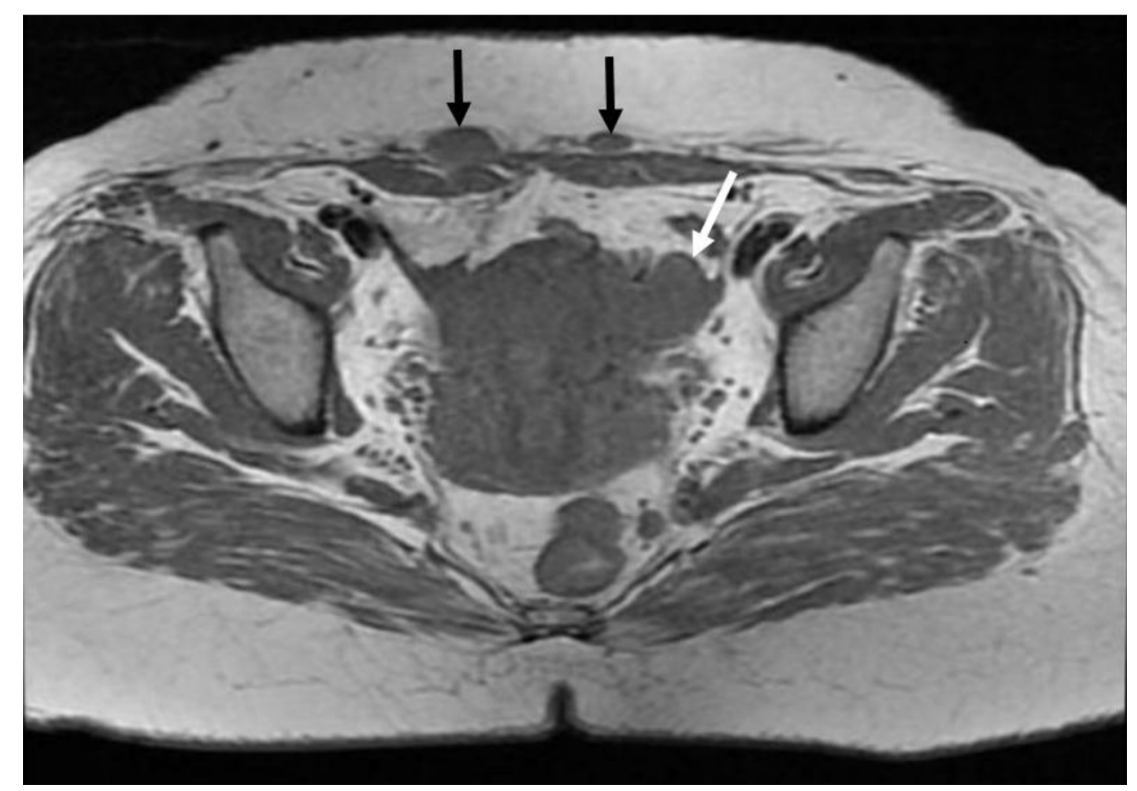

Figure 1. T1W image of the pelvis shows an Isosignal mass in the left ovary (white arrow). Note the soft tissue lesions in the right and left rectus abdominis muscles with similar signal intensity (black arrows).

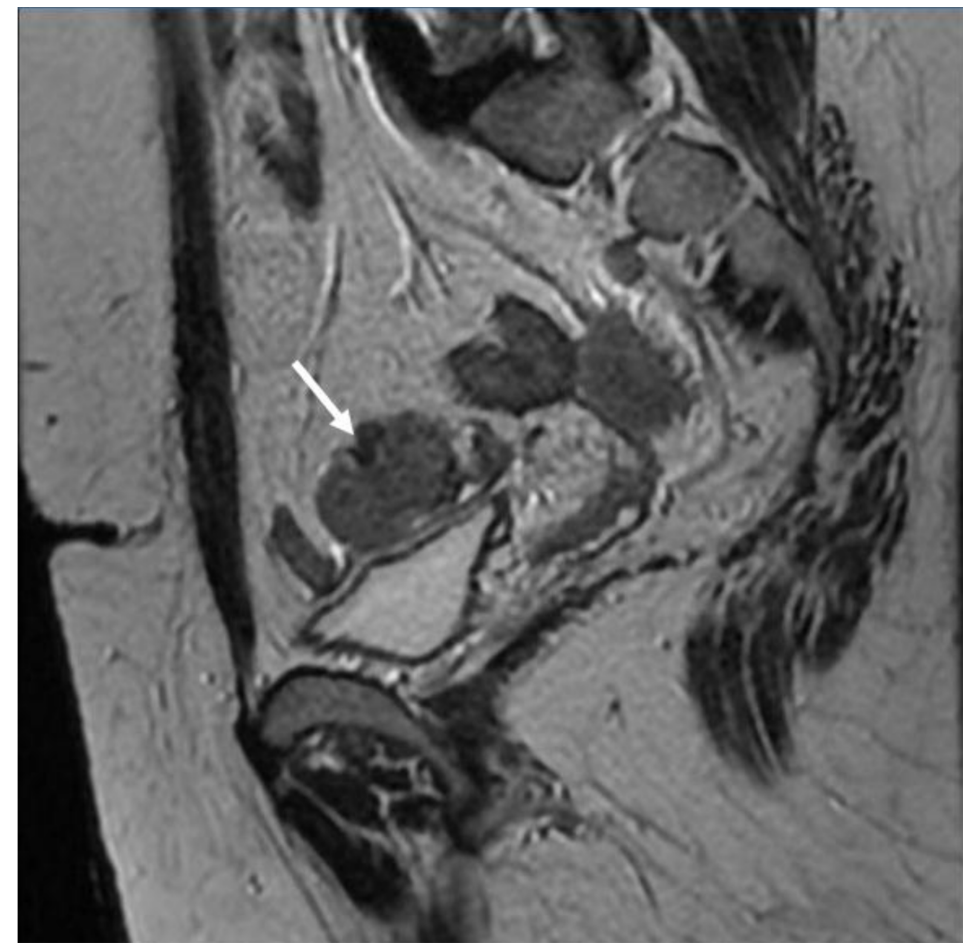

Figure 2. T2W sagittal image of the pelvis shows an iso to high signal intensity mass in the left ovary (white arrow). 


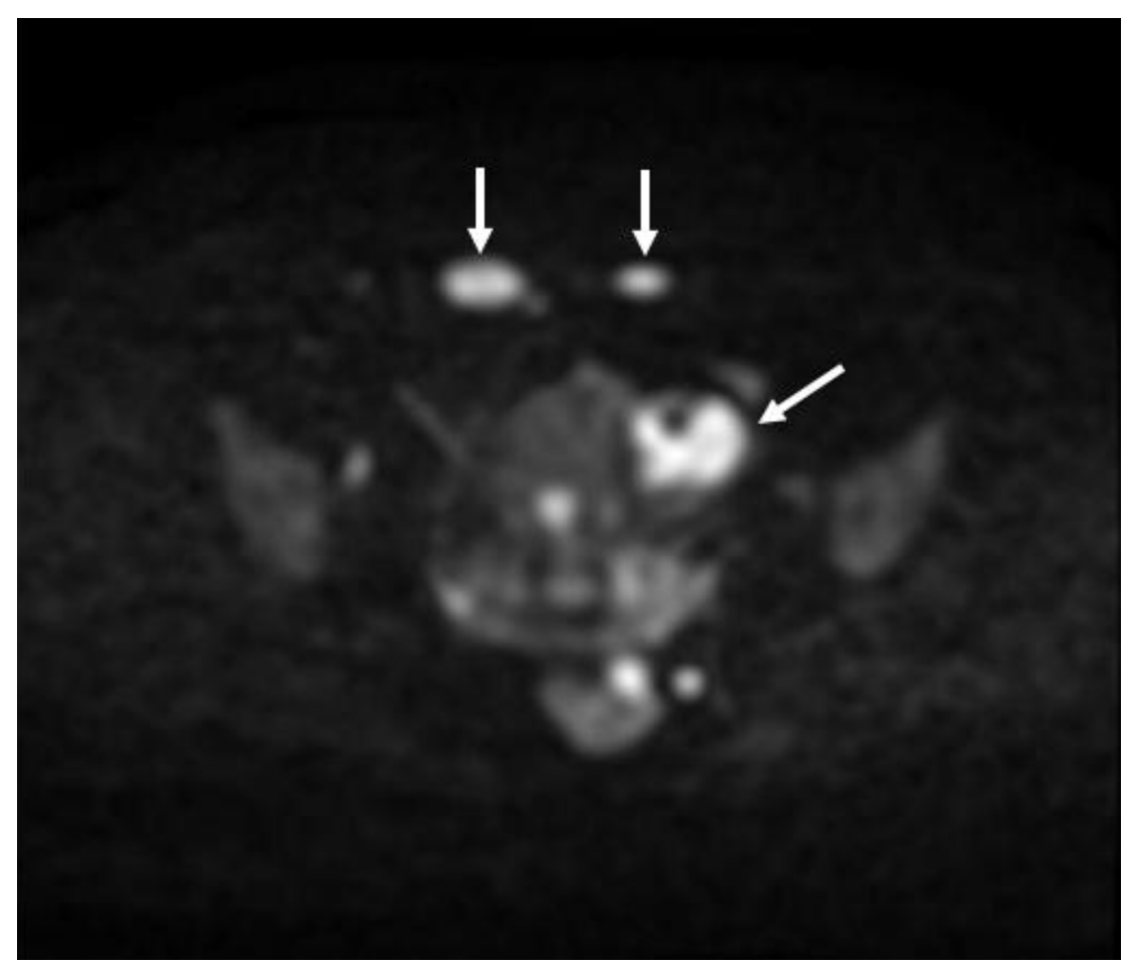

Figure 3. Diffusion-weighted imaging (DWI) shows a restricted diffusion pattern in both left ovarian mass and rectus abdominis muscles masses (white arrows).

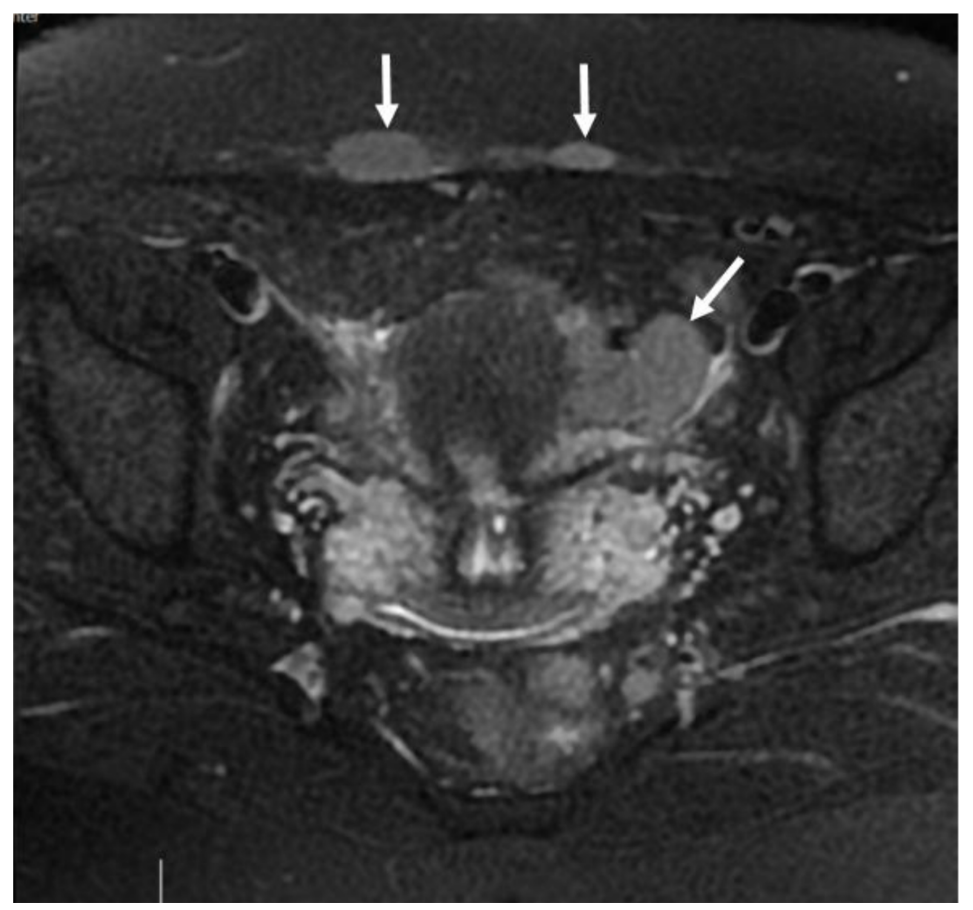

Figure 4. No signal suppression was found in both left ovarian mass and rectus abdominis muscles masses in T1 Fat suppression images (white arrows). 


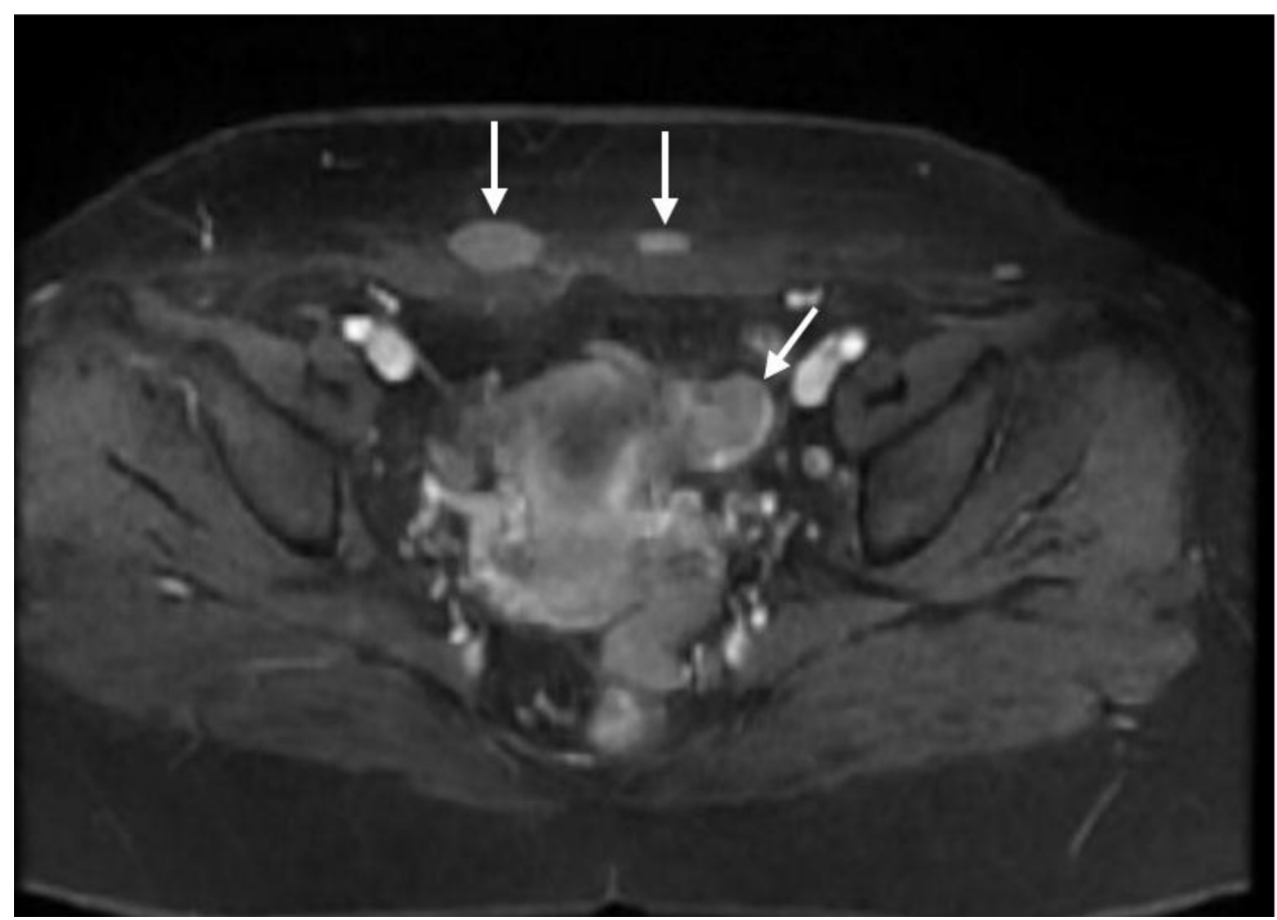

Figure 5. Lesions show homogenous enhancement in post-contrast MR images (white arrows).
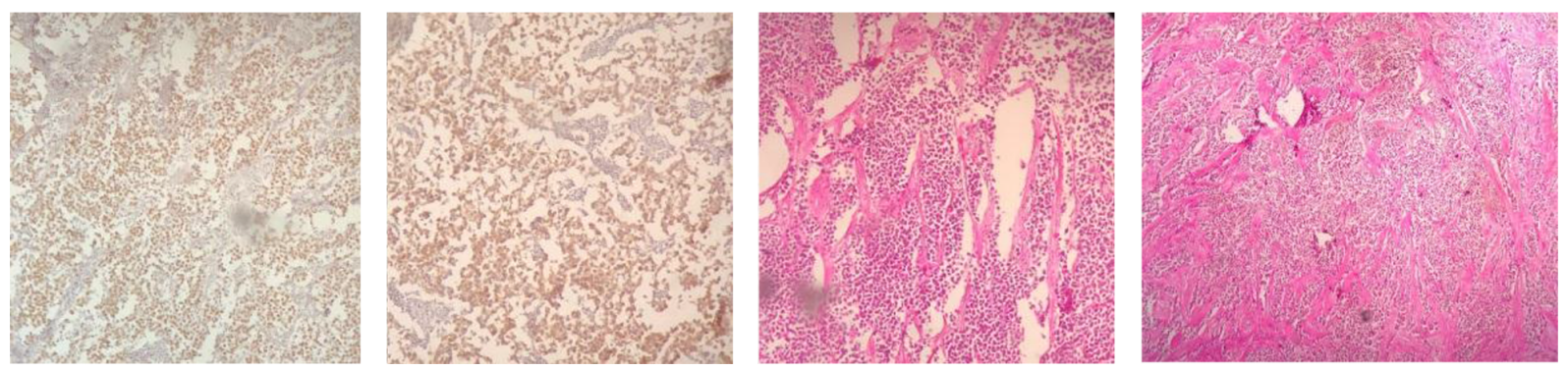

Figure 6. Sections show muscles and fibroconnective tissues with the invasive neoplastic lesion, composed of dispersed neoplastic cells by large atypical nuclei, nucleoli, and small acidophil cytoplasm enveloped without differentiation with chronic inflammatory hyalinized stroma.

points to be considered. Therefore, the clinician must examine the site of surgery in patients with history of malignancy and MRI can be utilized in the characterization of the skin lesions in these patients.

\section{Footnotes}

Authors' Contribution: Study concept and design: M.H.; Acquisition of data: A.T.; Analysis and interpretation of data: A.T.; Drafting of the manuscript: M.M.; Critical revision of the manuscript for important intellectual content:
A.T.; Administrative, technical, and material support: F.S.; Study supervision: M.H.

Conflict of Interests: The authors declare that there is no conflict of interest.

Funding/Support: This study was supported by Mashhad University of Medical Sciences.

Informed Consent: Written informed consent form provided by the patient for the publication and use of her images.. 


\section{References}

1. Hu SC, Chen GS, Wu CS, Chai CY, Chen WT, Lan CC. Rates of cutaneous metastases from different internal malignancies: experience from a Taiwanese medical center.JAm Acad Dermatol. 2009;60(3):379-87. doi: 10.1016/j.jaad.2008.10.007. [PubMed:19056145].

2. Lookingbill DP, Spangler N, Sexton F. Skin involvement as the presenting sign of internal carcinoma: : A retrospective study of 7316 cancer patients. J Am Acad Dermatol. 1990;22(1):19-26. doi: 10.1016/01909622(90)70002-y.

3. Ramirez PT, Wolf JK, Levenback C. Laparoscopic port-site metastases: etiology and prevention. Gynecol Oncol. 2003;91(1):179-89. doi: 10.1016/s0090-8258(03)00507-9.

4. Schwartz RA. Cutaneous metastatic disease. J Am Acad Dermatol. 1995;33(2):161-85. doi:10.1016/0190-9622(95)90231-7.

5. Bittencourt Mde J, Carvalho AH, Nascimento BA, Freitas LK, Parijos AM. Cutaneous metastasis of a breast cancer diagnosed 13 years before. An Bras Dermatol. 2015;90(3 Suppl 1):134-7. doi: 10.1590/abd18064841.20153842. [PubMed: 26312696]. [PubMed Central: PMC4540530].

6. Hsiu JENG-GWANG, Given Jr FT, Kemp GM. Tumor implantation after diagnostic laparoscopic biopsy of serous ovarian tumors of low malignant potential. Obstet Gynecol.1986;68(3 Suppl):90S-3S.

7. Alnafisah F, Dawa SK, Alalfy S. Skin Endometriosis at the Caesarean Section Scar: A Case Report and Review of the Literature. Cureus. 2018. doi: 10.7759/cureus.2063.

8. Otsuka I. Cutaneous Metastasis after Surgery, Injury, Lymphadenopathy, and Peritonitis: Possible Mechanisms. Int $\mathrm{J} \mathrm{Mol}$ Sci. 2019;20(13):3286. doi: 10.3390/ijms20133286. [PubMed: 31277406]. [PubMed Central: PMC6651228].

9. Weinberg D, Syed AA. Metastatic lung cancer presenting as cutaneous nodules. CMAJ. 2017;189(29). E966. doi: 10.1503/cmaj.161519. [PubMed:
28739849]. [PubMed Central: PMC5524578].

10. McDonald HH, Moore MR, Meffert JJ. Cutaneous metastases from adenocarcinoma of the ovary. JAAD Case Rep. 2016;2(5):406-7. doi: 10.1016/j.jdcr.2016.08.010. [PubMed: 27766303]. [PubMed Central: PMC5065639].

11. Adekunle OO, Zayyan M, Kolawole AO, Ahmed S. Case report: A rare case of dysgerminoma presenting with skin and breast metastasis. Clin Med Case Rep. 2013;2(2):170-2. doi:10.4236/crcm.2013.22046.

12. Sato Y, Hayashi T, Yamamoto H, Niina I, Kuroki N, Iwamura T, et al. Late Recurrence in Ovarian Dysgerminoma Presenting as a Primary Retroperitoneal Tumor: A Case Report and Review of the Literature. Case Rep Pathol. 2020;2020:4737606. doi: 10.1155/2020/4737606. [PubMed: 32110457]. [PubMed Central: PMC7042520].

13. Otsuka I. Cutaneous Metastases in Ovarian Cancer. Cancers (Basel). 2019;11(9):1292. doi: 10.3390/cancers11091292. [PubMed: 31480743]. [PubMed Central: PMC6788186].

14. Shah R, Xia C, Krailo M, Amatruda JF, Arul SG, Billmire DF, et al. Is carboplatin-based chemotherapy as effective as cisplatinbased chemotherapy in the treatment of advanced-stage dysgerminoma in children, adolescents and young adults? Gynecol Oncol. 2018;150(2):253-60. doi: 10.1016/j.ygyno.2018.05.025. [PubMed: 29884437].

15. Otsuka I, Matsuura T. Skin metastases in epithelial ovarian and fallopian tube carcinoma. Medicine (Baltimore). 2017;96(33). e7798. doi: 10.1097/MD.0000000000007798. [PubMed: 28816970]. [PubMed Central: PMC5571707].

16. Kadar N. Port-site recurrences following laparoscopic operations for gynaecological malignancies. Br J Obstet Gynaecol. 1997;104(11):130813. doi: 10.1111/j.1471-0528.1997.tb10980.x. [PubMed: 9386034]. 